\title{
Differences Between Sports Medicine in Europe and Japan
}

\author{
Yoshitomo SAITA*1) 2), Hiroshi IKEDA*1) 2), KAZUO KANEKO*1) 2) \\ *1) Department of Orthopedic Surgery and Sports Medicine, Juntendo University Hospital, Tokyo, Japan, \\ *2) FIFA Medical Centre of Excellence in Tokyo, Tokyo, Japan
}

\begin{abstract}
Sports medicine originates from Europe, and Japan is a developing country in this field. YS experienced medical support as a team physician, both in Japan and Europe. Several differences were observed between sports medicine in Europe and Japan. Firstly, the background of team physicians is different; team physicians in Japan are orthopedists, while they are sports physicians in Europe. Secondly, sports participants have to do periodic health evaluations before they start sports, even at amateur levels, but this is voluntary in Japan. Thirdly, surveillance of injuries and illnesses and injury prevention strategies are well-developed in Europe compared to Japan. Lastly, emergency systems are more advanced in Europe than Japan. Here, we describe these differences based on personal experience and a review of the literature.
\end{abstract}

Key words: sports medicine, Europe, Japan, pre-participation examination (PPE), injury survey

\section{Introduction}

The origins of sports medicine date back to ancient Greece and Rome. In modern times, the first international congress of sports medicine was held in 1928 at Switzerland, while the Japanese Society of Clinical Sports Medicine was established in 1989. Sports medicine has a longer history in European countries than in Japan. Furthermore, the organizations and facilities related to sports medicine are well established in Europe, while those in Japan are still being developed. Recently, there has been an increasing demand on sports medicine, not only from elite athletes, but also from recreational sports participants in Japan ${ }^{1)}$. Therefore, the development of high-level medical supports is required.

YS worked for more than ten years as an orthopedic surgeon at Juntendo Hospital, Japan. At the same time, YS worked as a team physician at a professional soccer club in Japan. Subsequently, YS was a clinical observer at the hospital of Istituto Ortopedico Galeazzi (Figure-1), Milan, Italy, from December 2015 to January 2017. This hospital performs 15,000 orthopedic surgical interventions per year. The center has 20 different functional units dedicated to Orthopedics, Traumatology and Joint replacement, and YS belonged to the Sports Traumatology and Arthroscopic Surgery unit. During the stay in Milan, YS also completed a clinical fellowship as a team physician in the medical department of AC Milan football club. AC Milan is a professional football (soccer) club based in Milan, Italy, that was founded in 1899 and is world-famous with several victories in the European Champions League (Figure-2). Therefore, YS experienced the medical support of professional football clubs as a team physician both in Japan and Europe, and the aim of this report is to compare the medical support systems between Europe and Japan.

Corresponding author: Yoshitomo Saita

Department of Orthopedic Surgery and Sports Medicine, Juntendo University Hospital

2-1-1 Hongo, Bunkyo-ku, Tokyo 113-8421, Japan

TEL: +81-3-3813-3111 E-mail: ysaita@juntendo.ac.jp

Special Reviews: 342nd Triannual Meeting of the Juntendo Medical Society “Medical Research Update” 〔Held on May 20, 2017〕

〔Received July 21, 2017〕〔Accepted Aug. 21, 2017〕

Copyright (C) 2018 The Juntendo Medical Society. This is an open access article distributed under the terms of Creative Commons Attribution License (CC BY), which permits unrestricted use, distribution, and reproduction in any medium, provided the original source is properly credited. doi: 10.14789/jmj.2018.64.JMJ17-R09 


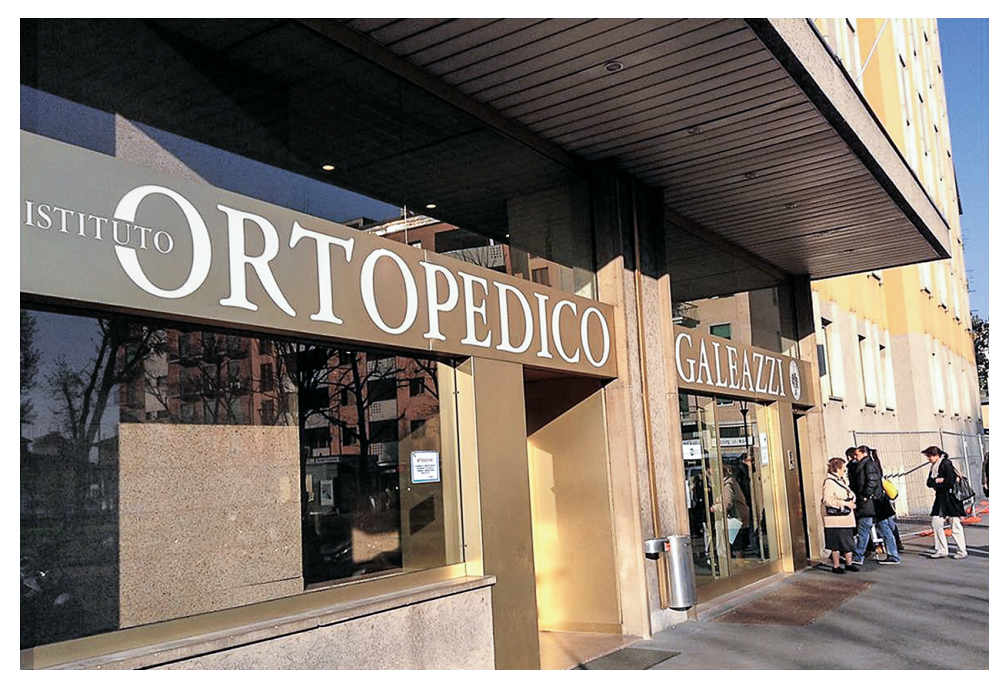

Figure-1 Istituto Ortopedico Galeazzi

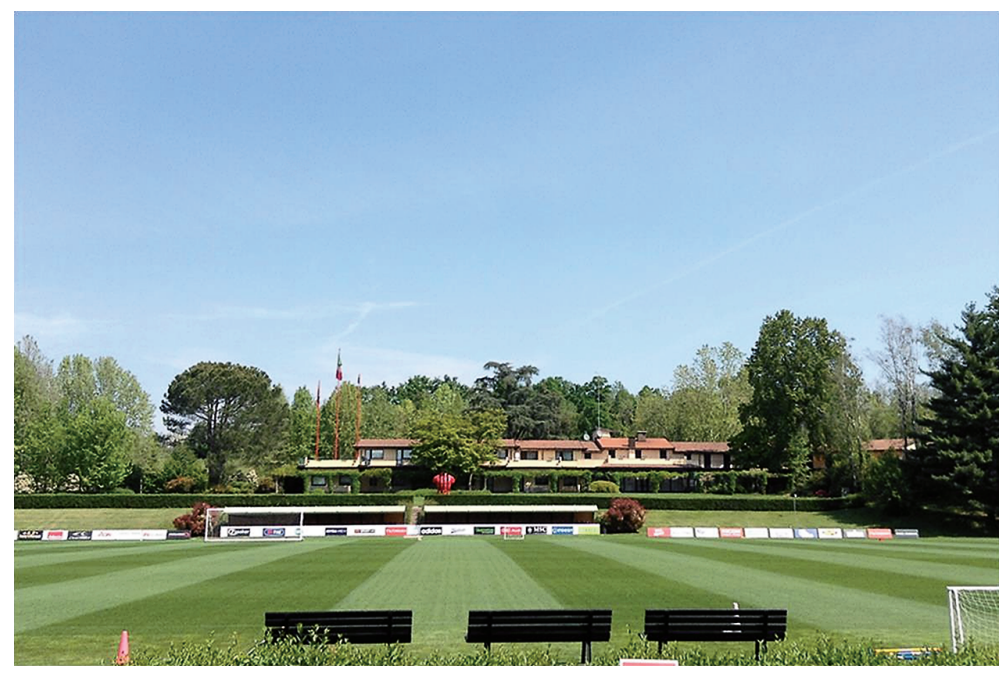

Figure-2 AC Milan Football Club (Milanello Sports Centre)

1. The different background specialty of team physicians in Japan and Europe

In Japan, the majority of team physicians are orthopedic surgeons. On the other hand, team physicians are "sports doctors" in Europe. The latter have studied special curricula for at least five years and passed examinations to be sports doctors. They are not surgeons, but generalists familiar with almost all the field of sports medicine, including internal medicine, sports traumatology, nutrition, conditioning, training methodologies, and so on. At professional football (soccer) clubs in Italy, most team physicians stay in the club house while players are performing exercises or undergoing treatments. If a player injured their knee joint, a team physician performs the primary treatment and physical examination. They judge whether injured players need any further examinations, such as radiographic evaluations, and then coordinates visits to specialists such as knee surgeons. If the injured player needs to undergo surgical treatment, specialists working at hospitals perform the surgery. Therefore, team physicians don' $t$ normally perform surgical treatments in Italy. By contrast, in Japan, team physicians work at hospitals and perform the surgery themselves if a player requires treatment.

The system in Japan has the advantage that players undergo surgery by the doctor who knows them well before injury, and can easily communicate with other support staff on the condition of players and rehabilitation plans. However, this system also possesses several disadvantages. Firstly, Japanese orthopedists are familiar with 


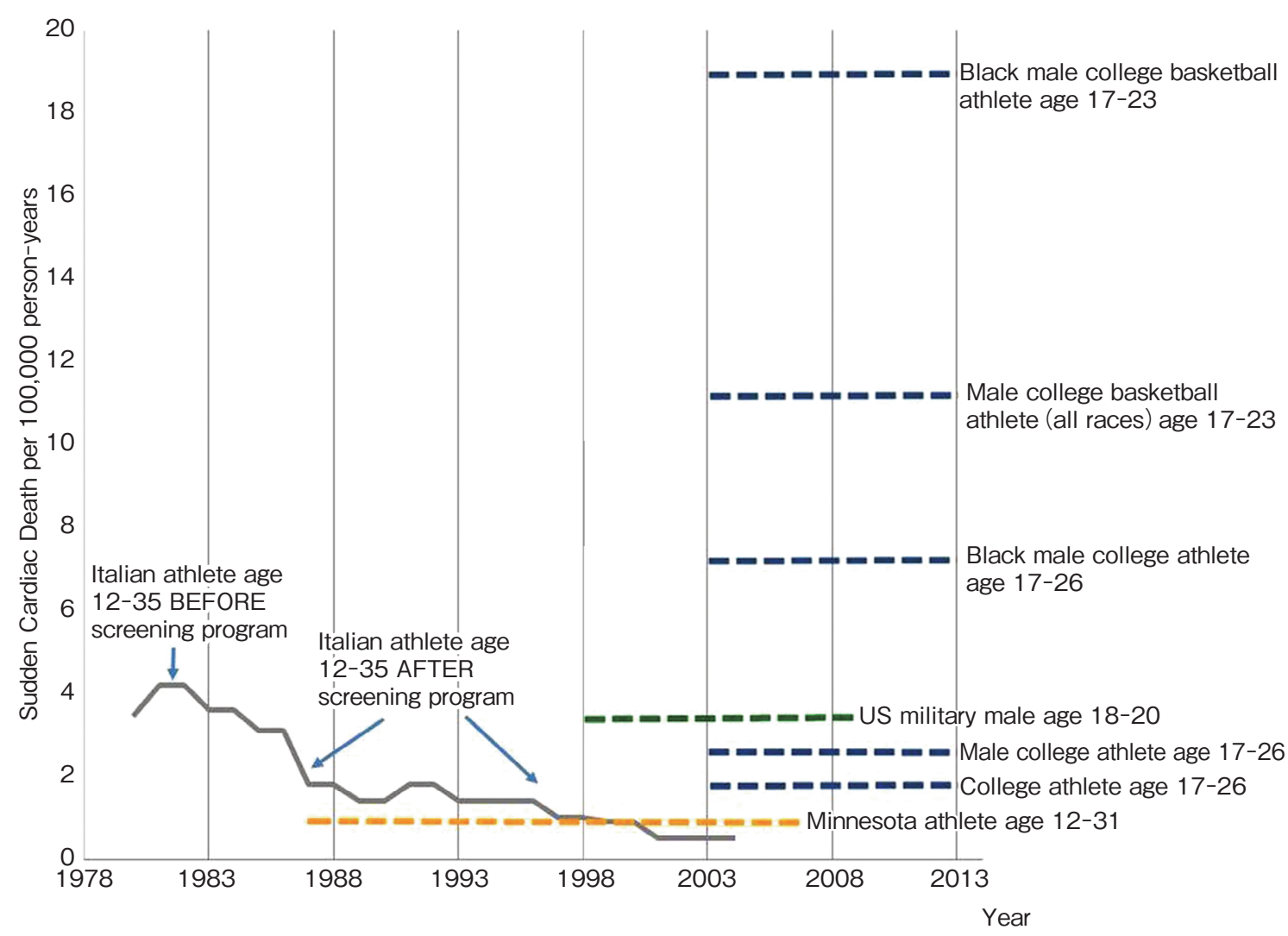

Figure-3

The risk of Sudden Cardiac Death (SCD) and sports ${ }^{2}$. Annual risk of SCD in athletes from Veneto, Italy ${ }^{3)}$ and Minnesota ${ }^{4)}$, and more recent incidence data in NCAA college athletes ${ }^{5)}$ and US military personnel ${ }^{6)}$. The differential risk of SCD in targeted athlete populations should be considered when choosing an appropriate CV screening strategy. Graph adapted from Corrado et al. ${ }^{3)}$. CV, cardiovascular; SCD, sudden cardiac death.

(Drezner JA, et al: Br J Sports Med, 2016; 50: 1376-1378 ${ }^{2}$. Corrado D, et al: JAMA, 2006; 296: 1593-1601 3). Harmon KG, et al: Circulation, 2015; 132: 10-194). Maron BJ,et al: Am J Cardiol, 2009; 104: 276-280 ${ }^{5)}$. Eckart RE, et al: J Am Coll Cardiol, 2011; 58: $1254-1261^{6)}$ )

sports traumatology, but often lack education in other fields of sports medicine. They tend to focus on how to "treat injured players" but pay a less attention to the total health care of athletes and prevention of injuries. Secondly, when the team doctor performs a surgery and the prognosis is poor, the team doctor runs the risk of losing the trust of players and team staff. In a worst-case situation, they are unable to continue their work as a team physician.

In Italy, the situation is different from Japan. They have both generalists (team physician) and specialists (radiologists, orthopedists, phycologists, and so on). However, this system also possesses a drawback. Although team doctors may not be familiar with the details of surgical interventions, they must decide treatment strategies for players, and many specialists offer differing opinions to the team doctor. In this situation, team doctors usually follow the opinion of the specialists, even though they may have a different opinion, because they have a risk of losing the trust of players if they fail to recover well. As a result, Italian team doctors tend to avoid making decisions when they manage players.

Although medical support systems in Italy are well established and have a much longer history than Japan, there also exists unfavorable aspects as described above. In Japan, we should keep these facts in mind when we improve sports medical support systems, leading to the development of original systems suitable for Japanese culture.

\section{Importance of Pre-Participation Examination (PPE)}

Federazione Italiana Giuoco Calcio (FIGC, Italian Football Federation) recommends a periodic health evaluation (PHE) for all players which are 


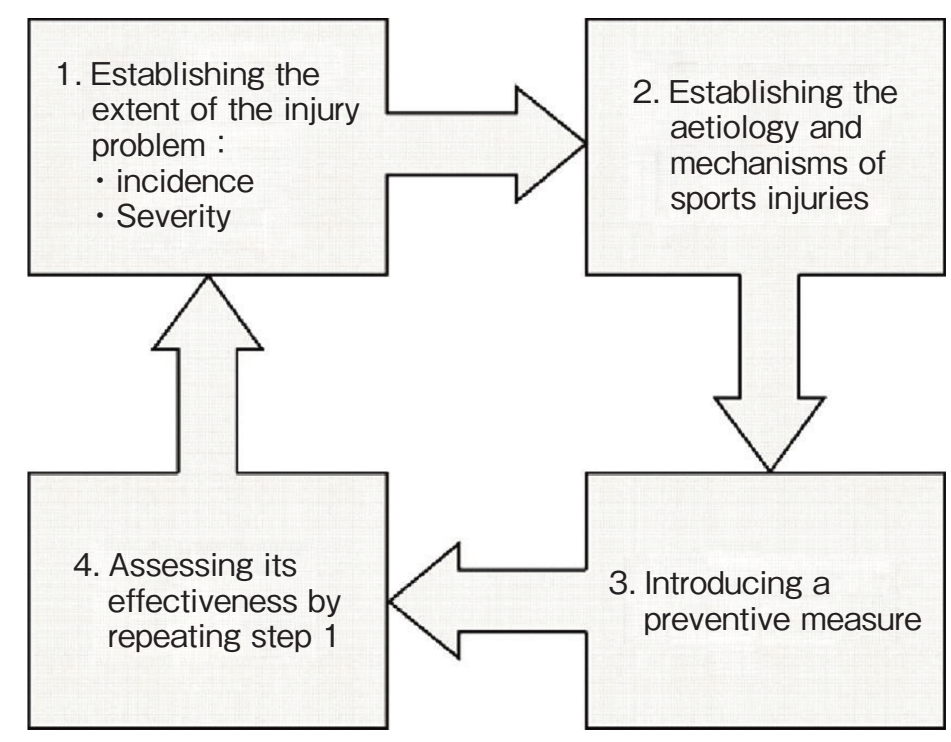

Figure-4

Four-step sequence of injury prevention. van Mechelen's four-step injury prevention model. Incidence, severity, aetiology and prevention of sports injuries. A review of concepts. ${ }^{7)}$ (van Mechelen W, et al: Sports Med, 1992; 14: 82-997)

performed by a sports physician. They impose PreParticipation Examination (PPE) prior to engagement in competitive sport. It is the responsibility of the National Federations to ensure that these pre-competition health screening procedures have been performed. The FIGC are required to confirm the PPE Certification that all players must complete, and the certificate should be sent to FIGC prior to registration. The main purpose of this screening is to identify athletes at risk of Sudden Cardiac Death (SCDs). According to the European Society of Cardiology (ESC), epidemiological studies on thousands of competitive athletes showed a decrease of up to $89 \%$ in SCDs with a PPE including a 12-lead rest echocardiogram. Data from the Veneto region of Italy show that the incidence of sudden death in young athletes fell from 3.6 per 100,000 person-years to 0.4 per 100,000 personyears after imposing PPE on athletes (Figure-3) ${ }^{2-6)}$. They emphasized the importance of cardiovascular screening in young athletes. Therefore, FIGC imposes PPE to all players, even in primary school children. In Japan, however, there are no such screening systems for young athletes. Surprisingly, even the incidence of SCDs in Japanese athletes is unknown because there are no national surveillances programs for sports-related illnesses in Japan. Therefore, in terms of medical screening systems for sports participants and sports-related illness surveillance, Japan can be considered an underdeveloped country. Considering the global standard of sports medicine, we require a concerted effort to establish systems with PPE and illness surveillance in Japan as soon as possible.

\section{Injury prevention strategies in Europe and Japan}

The four-step injury prevention model of van Mechelen is the world-wide gold standard $(\text { Figure }-4)^{7}$. In order to develop an effective injury prevention program, we should always consider the following questions. What is known about the size of the problem (step 1)? What is known about risk factors (step 2)? What is known about the effectiveness of preventive measures (step 3)? Are there systems in place to monitor sports injury (step 4)?

The first step for injury prevention is to "know the problem", and this can be achieved through "injury survey". In 2001, Union of European Football Associations (UEFA) initiated a research program with the aim of increasing the safety of players in its competitions and contributing to the wider understanding of injury in sport. This project, the "UEFA Elite Club Injury Study", has been continuing for more than 15 years with elite clubs in the UEFA Champions League, including AC Milan. In 2011, the leaders of this group, Jan Ekstrand et $a l$., analyzed the data of this UEFA Champions 


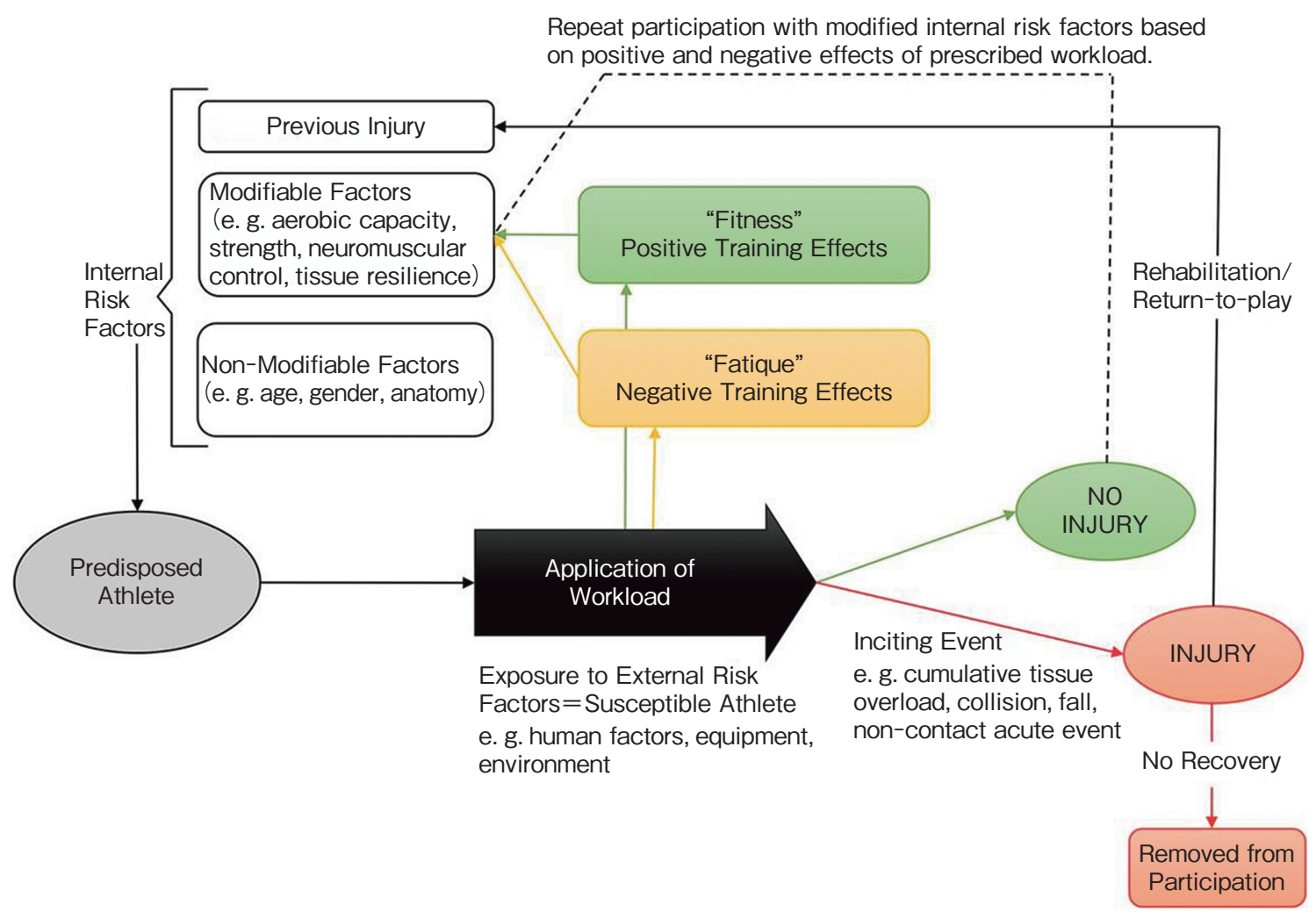

Figure-5 The workload-injury aetiology model

How do training and competition workloads relate to injury? Windt \& Gabbett proposed the workload-injury aetiology model ${ }^{9)}$.

(Windt J, et al: Br J Sports Med, 2017; 51: 428-435. ${ }^{9)}$ )

League injury survey from 2001 to 2008. The database included 23 teams, 4,483 injuries, 566,000 hours of exposure ${ }^{8)}$. This prospective cohort study generated a large amount of important information regarding the incidence and situation of the occurrence of sports injuries, such as muscle strain, ligament injury, concussion, and stress fracture. The results of this study greatly contributed to European sports physicians progressing their strategies for injury prevention. Recently, Windt and Gabbett proposed an updated injury aetiology model that explicitly incorporates workloads within the causal chain, and outlines its known effects $(\text { Figure }-5)^{9)}$. According to this model, internal risk factors are differentiated into modifiable and non-modifiable factors, and workloads contribute to injury in three ways: 1; exposure to external risk factors and potential inciting events, 2; fatigue, or negative physiological effects, and 3; fitness, or positive physiological adaptations. Exposure is determined solely by total load, while positive and negative adaptations are controlled both by total workloads, as well as changes in load.

These studies inform us that, in European
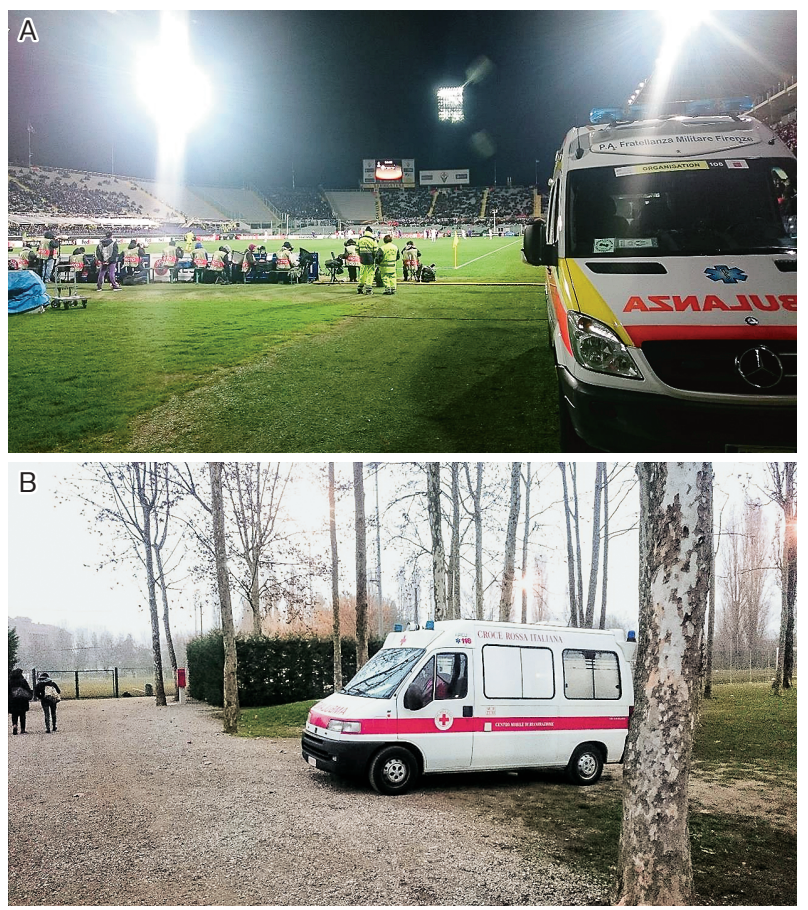

Figure-6

A: Ambulance and emergency life-saving technician on the stadium (Serie A). B: Ambulance and emergency life-saving technician beside the pitch (under 15). 
countries, the four-step injury prevention model (Figure-3) has been successful. On the other hand, in Japan, most sports doctors are orthopedists working in hospitals, and it is difficult for them to be involved in the injury prevention of athletes. Furthermore, there is no systematic educational system to become a sports doctor, there are no injury surveillance systems similar to the UEFA injury survey. We must consider these facts and take action to improve this situation in order to protect athletes from injury and raise players' performance. To achieve these goals, it seems that cooperation between industry, government, and academia will be important.

\section{The differences between emergency medical systems in Europe and Japan}

In football stadia in Europe, the hosts of the football games have to prepare for emergency situations, not only for the serious injury of athletes, but also illnesses and injuries among spectators. In addition, there is growing awareness of the need to be prepared for the possibility of a terrorist attack. Therefore, in Italy, FIGC require that ambulance and emergency life-saving technicians have to stay in the stadium during all official games, from under 15 categories to professional league (Serie A) (Figure-6). By contrast, in Japan, ambulance and life-saving technicians cannot be on standby at the stadium due to fire laws, even in the Japanese professional football league (https://www.jleague. jp/aboutj/regulation/). In 2014 in Japan, it took 7 minutes and 54 seconds on average for an ambulance to arrive on a scene. According to the Fire and Disaster Management Agency (FDMA) of Japan, the average time from calling 119, Japan's emergency telephone number, to arriving at a hospital was about 39 minutes nationwide in the fiscal year 2013, which is about 10 minutes longer than a decade earlier. This is a consequence of an increasing number of ambulance calls, with more ambulances having to be dispatched from distant fire departments. In this background, ambulances cannot be on standby at stadia in Japan. However, in the developed countries that host international sports events, such as The FIFA World Cup and the Olympic Games, it is common sense that the host must ensure sufficient emergency care systems for athletes and spectators. Therefore, as with injury survey, cooperation between industry (including private ambulance services), government (FDMA), and academia (universities) will be important for developing emergency medical support systems in Japan.

\section{Acknowledgement}

YS would like to express his sincere gratitude to Juntendo University Graduates' Association for their financial support that made it possible for him to continue studying in Italy with his family.

\section{References}

1) Okamatsu H: Athletic trainers are needed for athletic activities in Japan RADIANT Ritsumeikan University Research Report, 2016, Issue 3.

2) Drezner JA, Harmon KG, Asif IM, Marek JC: Why cardiovascular screening in young athletes can save lives: a critical review. Br J Sports Med, 2016; 50: 13761378.

3) Corrado D, Basso C, Pavei A, Michieli P, Schiavon M, Thiene G: Trends in sudden cardiovascular death in young competitive athletes after implementation of a preparticipation screening program. JAMA, 2006; 296: 1593-1601.

4) Harmon KG, Asif IM, Maleszewski JJ, et al: Incidence, cause, and comparative frequency of sudden cardiac death in national collegiate athletic association athletes: a decade in review. Circulation, 2015; 132: 10-19.

5) Maron BJ, Haas TS, Doerer JJ, Thompson PD, Hodges JS: Comparison of U.S. and Italian experiences with sudden cardiac deaths in young competitive athletes and implications for preparticipation screening strategies. Am J Cardiol, 2009; 104: 276-280.

6) Eckart RE, Shry EA, Burke AP, et al; Department of Defense Cardiovascular Death Registry Group: Sudden death in young adults an autopsy-based series of a population undergoing active surveillance. J Am Coll Cardiol, 2011; 58: 1254-1261.

7) van Mechelen W, Hlobil H, Kemper HC: Incidence, severity, aetiology and prevention of sports injuries. A review of concepts. Sports Med, 1992; 14: 82-99.

8) Ekstrand J, Hägglund M, Waldén M: Injury incidence and injury patterns in professional football: the UEFA injury study. Br J Sports Med, 2011; 45: 553-558.

9) Windt J, Gabbett TJ: How do training and competition workloads relate to injury? The workload-injury aetiology model. Br J Sports Med, 2017; 51: 428-435. 